\title{
Impacto del Programa TARGA en la disminución de casos de sida en el sistema de salud peruano, 1983-2018
}

\author{
Yordanis Enríquez Canto, ${ }^{1}$ Giovani Martín Díaz Gervasi ${ }^{1}$ y Luis Alberto Menacho Alvirio ${ }^{2}$
}

Forma de citar Enríquez Canto Y, Díaz Gervasi GM, Menacho Alvirio LA. Impacto del Programa TARGA en el sistema de salud peruano en la disminución de casos de sida, 1983-2018. Rev Panam Salud Publica. 2020;44:e27. https://doi.org/10.26633/RPSP.2020.27

RESUMEN

Objetivo. Evaluar el impacto del programa de terapia antirretroviral de gran actividad (TARGA) en la disminución de casos estadio sida en Perú en el período 1983-2018.

Métodos. Experimento natural con diseño de series de tiempo interrumpidas durante un período de 36 años. Se analizaron los casos de sida según el año de diagnóstico y la política de acceso universal a la terapia con antirretrovirales del año 2004. Se emplearon datos agregados del Centro Nacional de Epidemiología, Prevención y Control de Enfermedades de 1983 al 2018. Se utilizó la regresión logarítmica lineal y se evaluó el Programa TARGA con un modelo autorregresivo integrado de media móvil (ARIMA, por sus siglas en inglés), a fin de realizar un pronóstico del número esperado de casos anuales de sida, y mediante series de tiempo interrumpidas.

Resultados. Entre 2004 y 2018 hubo un decremento anual de 2,60\% de casos (intervalo de confianza del 95\% [IC95\%]: -3,9 - 1,2). El mejor modelo ARIMA identificado tuvo los componentes: (8, 3, 1). El pronóstico arrojó un total de 32709 nuevos casos de sida para este período, con una prevención estimada de 10280 casos. El análisis de series de tiempo arrojó una disminución anual de 134,21 (IC 95\%: -160 - -108,60) casos. Conclusiones. Los hallazgos evidenciaron un aumento sustancial en los casos de sida durante el período 1983-2004, con una reducción significativa posterior a la implementación del Programa TARGA. El programa se confirma como una intervención efectiva para la prevención del número de casos de sida, permite el acceso universal a la terapia con antirretrovirales y otorga otros servicios como pruebas de monitoreo, consejería psicológica y el fomento de la adherencia al tratamiento en las personas que viven con VIH-sida.

Palabras clave Política de salud; terapia antirretroviral altamente activa; síndrome de inmunodeficiencia adquirida; sistemas de salud; Perú.

En Perú se trabaja en el control de la epidemia del virus de inmunodeficiencia humana (VIH) mediante estrategias de prevención e intervenciones biomédicas (1-3). En época reciente, se ha centrado la atención en la expansión del tratamiento contra el VIH para frenar su progresión hacia la fase de sida $(4,5)$. La terapia antirretroviral de gran actividad (TARGA) suprime la replicación del VIH de forma sostenida, lo que conduce a una carga viral indetectable, con disminución de la morbilidad y la mortalidad $(6,7)$.

Aproximadamente $4 \%$ de las personas que viven con $\mathrm{VIH} /$ sida (PVVS) en América Latina se encuentran en Perú (8), con

\footnotetext{
Facultad de Ciencias de la Salud Universidad Católica Sedes Sapientiae, Lima, Perú. $₫$ Yordanis Enríquez Canto, yenriquez@ucss.edu.pe
}

43072 casos de sida acumulados hasta el 2018 (9). El escenario peruano de $\mathrm{VIH} /$ sida se concentra en grupos clave y se transmite sobre todo por vía sexual $(97,6 \%)(8)$. La prevalencia del VIH fue de $0,3 \%$ en adultos en el 2018. Sin embargo, es mayor entre los hombres que tienen sexo con hombres $(15,2 \%)$, las personas transgénero $(13,8 \%)$ y trabajadoras sexuales $(1,3 \%)(9,10)$. Se estima que el número de casos nuevos de $\mathrm{VIH}$ reportados entre 2017 (5 926) y 2018 (5 911) se mantiene estable (9). Por otra parte, el número de casos de sida disminuyó de 2254 en el 2005 a 1362 en el 2018, con una razón hombre/mujer de 4,5 (9). Por su parte, el número de muertes por sida fue de 2200 casos en el

\footnotetext{
2 Facultad de Salud Pública y Administración, Universidad Peruana Cayetano Heredia, Lima, Perú.
} 
2016 (10). En América Latina, la mayor reducción de mortalidad por sida se observa en el Perú, con una disminución de $62 \%$ entre el 2000-2016 (10).

En el Perú, el Programa Nacional de Terapia Antirretroviral de Gran Actividad (Programa TARGA) comenzó el 13 de mayo del 2004. Este se desarrolló a través del Ministerio de Salud del Perú (MINSA) y la Estrategia Sanitaria Nacional (11). $\mathrm{Al}$ inicio, se implementó en 15 hospitales de Lima y Callao, y en 33 regionales. Se concentró en la capacitación al personal de salud, en asegurar la disponibilidad de la medicación y la adherencia a la misma $(12,13)$. En el programa se brindan, de manera gratuita, el tratamiento antirretroviral (TARV), las pruebas de monitoreo y la consejería psicológica relacionada a la disminución de prácticas sexuales de riesgo y adherencia al tratamiento.

La implementación del Programa TARGA estuvo complementada por la norma técnica del 2004. Uno de los criterios para iniciar TARV fue tener un recuento de linfocitos T CD4 menor de 200 células $/ \mathrm{mm}^{3}$ (14). En los primeros dos años, se reportó un incremento en la tasa de mortalidad por inicio tardío del tratamiento. Sesenta y tres por ciento de los pacientes iniciaba tratamiento con un CD4 menor de 100 células $/ \mathrm{mm}^{3}$, mientras que $66 \%$ lo hacía con una carga viral mayor a 100000 copias / $\mathrm{mL}$ (13). El inicio de TARV en un estadio avanzado se asocia con una mayor toxicidad medicamentosa y con el síndrome de reconstitución inmune (14). Sin embargo, el inicio del tratamiento se ha adelantado de manera progresiva, con recuentos cada vez más altos de T CD4 (350 y 500 células $/ \mathrm{mm}^{3}$ ), lo que reduce el riesgo de progresión de la enfermedad $(15,16)$. Desde el 2016, la OMS recomienda el tratamiento universal a todas las personas con VIH cualquiera sea el recuento de CD4 (17). Este enfoque de inicio inmediato del tratamiento es propuesto en la última norma técnica peruana del 2018 (18).

El impacto de una política de salud pública depende de su implementación (19). En este sentido, las mejoras en el Programa TARGA se han llevado a cabo mediante el refuerzo de la estrategia sanitaria nacional y asumiendo los costos de la terapia. Este compromiso se refleja en la reducción de los casos nuevos de sida y en el aumento de la cantidad de centros de atención al VIH. En el 2018, se reportaron 152 centros en comparación con los 86 que existían en el 2011 (9). Como resultado, la cobertura de TARV se incrementó de 25\% (2011) a 68\% (2017) (9). Sin embargo, aún existen brechas importantes en el continuo de atención con base en los objetivos 90-90-90 del Programa Conjunto de las Naciones Unidas sobre el VIH/sida (ONUSIDA): $90 \%$ de personas diagnosticadas, $90 \%$ de personas en tratamiento y $90 \%$ con carga viral indetectable es la meta fijada para el año 2020 con el objeto de controlar y erradicar el virus (20).

Perú se ha sumado a la pauta de expansión global de TARV en el marco del tratamiento del VIH como prevención. Sin embargo, a nivel nacional, aun no se ha evaluado en detalle el efecto en la reducción de casos de sida del Programa TARGA, que prevé el acceso gratuito y universal. Es importante estimar su contribución a fin de establecer nuevos programas en forma adecuada. De esta manera, se buscó evaluar el impacto de la incorporación del Programa TARGA al sistema de salud peruano (SSP) en la disminución de los casos estadio sida reportados entre 1983 y 2018.

\section{MATERIALES Y MÉTODOS}

Se trata de un experimento natural (EN) con diseño de series de tiempo interrumpidas (STI) (21). En los EN, las condiciones experimentales y de control de los individuos expuestos no están determinadas por los investigadores, ya que estos no tienen control sobre la intervención (21). Cuando la experimentación controlada no es factible de implementar o no es ética, los EN se emplean como diseños de estudio (21). En este sentido, la intervención estudiada fue el programa de salud que permite el acceso universal a TARV implementado en Perú. No fue posible realizar asignación al azar, por lo que se empleó el enfoque de STI (22). Este es considerado, en tales contextos, como un diseño cuasiexperimental sólido y de aceptada utilización en evaluaciones de intervenciones de salud pública (22).

En el estudio se emplearon series temporales de datos agregados del Centro Nacional de Epidemiología, Prevención y Control de Enfermedades (CDC). Los datos consolidados a nivel nacional se recolectan y presentan todos los años. Esta información proviene de la Red Nacional de Vigilancia Epidemiológica, así como de las fuentes declarantes del Sistema. Se consideraron series del 1983 al 2018.

La variable dependiente fueron los casos notificados a nivel nacional de VIH en estadio sida por año de diagnóstico según la definición de caso de acuerdo con criterios clínicos y de laboratorio $(13,18)$, con base en un recuento bajo de CD4 (estadio 3) y la presencia de una enfermedad oportunista (estadio C) (18).

Se calcularon medias, frecuencias y porcentajes de la variable dependiente. Para el análisis de tendencias de los casos notificados de sida, se utilizó la regresión logarítmica lineal con punto de unión (joinpoint). Los parámetros resultantes del análisis fueron: el cambio porcentual anual (CPA) estimado de la variable dependiente para cada período y el cambio porcentual anual promedio del período (AAPC). Se buscó un máximo de cinco puntos de inflexión y se utilizó el software Joinpoint Regression Program 4.7.0.0 $0^{\circledR}$.

La serie de tiempo analizada fue de 36 años. A fin de evitar los problemas de autocorrelación que conllevan a la sobreestimación de la significación estadística del efecto estimado de la intervención, se empleó un modelo autorregresivo integrado de media móvil (ARIMA) (23). El modelo óptimo puede incluir componentes de autorregresión, componentes de media móvil y operaciones de diferenciación de la serie. Dichos términos se denominan $\mathrm{p}, \mathrm{d}$ y $\mathrm{q}$, respectivamente; donde $\mathrm{p}$ representa el mayor número de rezagos del parámetro autorregresivo, $\mathrm{d}$ es el grado de diferenciación de la serie, y q es el mayor número de componentes de la media móvil (23).

El efecto del Programa TARGA, entonces, se evaluó mediante un modelo ARIMA a fin de realizar un pronóstico del número esperado de casos anuales de sida como si el programa no se hubiese puesto en práctica. Este número se comparó con los casos anuales reportados de sida luego de su introducción. Se aplicó la prueba Dickey-Fuller para determinar la presencia de estacionariedad en la serie. También, se analizó una STI evaluándose tendencias y cambios inmediatos significativos atribuibles a la introducción de TARV con el enfoque de mínimos cuadrados lineales. Se estimaron coeficientes produciendo errores estándares Newey-West para manejar la autocorrelación además de la posible heterocedasticidad. Se empleó el software Stata $14^{\circledR}$ y se consideraron intervalos de confianza al $95 \%$. 
El Comité de ética de la Universidad Católica Sedes Sapientiae aprobó el estudio considerándolo como análisis de información pública agregada. Los datos de los participantes se encontraron anonimizados en la fuente (CDC).

\section{RESULTADOS}

En el Perú, se reportaron 40551 casos nuevos de VIH en estadio sida desde 1983 al 2018. El período preintervención abarcó 22 años (1983-2004), mientras que fueron 14 los años posteriores a la introducción de TARV (2005-2018). Los casos anuales anteriores a la implementación de TARV fueron 18 122. Por su parte, se reportaron 22429 nuevos casos en el período posintroducción. En la figura 1 se observa un decremento de casos luego de la puesta en vigor del programa.

En el cuadro 1 se detallan las tendencias para los casos anuales notificados. Para el período analizado de 36 años, resultaron cinco segmentos con significancia estadística. Entre ellos, el segmento 1985-1988 con un incremento anual de 317,09\% (IC 95\%: 209,8 - 461,6). Para el segmento 2004-2018, el CPA reportó un decremento anual de 260\% (IC 95\%: -3,9 - 1,2) en los casos estadio sida. Sin embargo, el AAPC para el período estudiado fue de $22,3 \%$ (IC 95\%: 17,3 - 27,5).

La inspección gráfica de la serie de tiempo señaló la presencia de tendencia, así que se procedió a verificar la estacionariedad y a su posterior diferenciación. Se realizó la prueba Dickey-Fuller de la tercera diferencia $(p, 3, q)(P=0,0070)$ por lo que se rechazó la hipótesis nula de presencia de raíz unitaria. A continuación, la inspección de los autocorrelogramas total y parcial de la tercera diferencia de la serie indicó del componente autorregresivo 8 rezagos $(8,3, q)(P=0,041)$. El número de elementos de la media móvil fue $1(P=0,004)$. Entre los posibles candidatos el mejor modelo identificado tuvo los componentes: $(8,3$, 1). Los parámetros considerados para su evaluación fueron el logaritmo de verosimilitud más alto $(-223,95)$, y el criterio informativo de Akaike, más bajo $(467,90)$.

A partir del análisis ARIMA univariante, ajustado para los datos de la preinstalación de TARV, se desarrolló un pronóstico de casos esperados para los 14 años siguientes a la misma. La predicción reflejó los casos que pudieran haber sido notificados, en caso de no haber tenido acceso al Programa TARGA. Entre el 2005 y el 2018 se reportó un total de 22429 casos. El pronóstico arrojó un total de 32709 casos nuevos de sida para este período. En consecuencia, el programa resultó en la prevención estimada de 10280 casos nuevos de sida, con una media anual de 734 casos. En la figura 2 se aprecia la contrastación de los casos observados y los pronosticados.

Luego, se aplicó un diseño de STI para evaluar el efecto del Programa TARGA. Se observaron cambios estadísticamente significativos en la pendiente respecto a la cantidad casos de sida en la población peruana después de 2004 (figura 3). Los coeficientes para el cambio de nivel y de pendiente en la serie se presentan en el cuadro 2. En el análisis de STI los cambios de nivel representan una disminución (coeficientes negativos) o un aumento (coeficientes positivos) en la cantidad de casos de SIDA reportados, mientras que los coeficientes de cambio

FIGURA 1. Casos anuales de VIH en estadio de sida (1983-2018)

2500

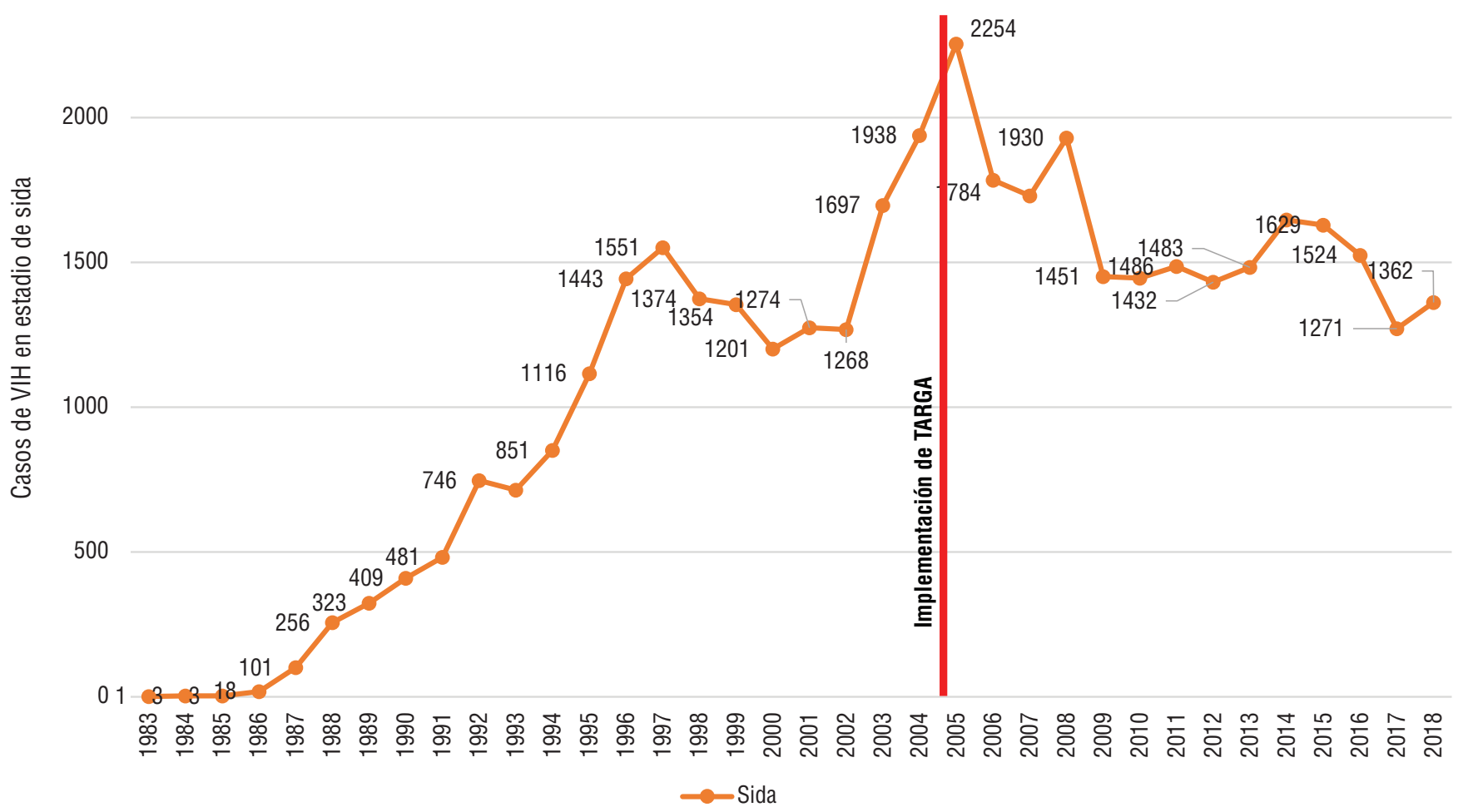

Fuente: elaboración propia a partir de los resultados del estudio 
CUADRO 1. Tendencias de los casos notificados de VIH en estadio sida según el año de diagnóstico en Perú (1983-2018), análisis de regresión lineal segmentada

\begin{tabular}{lcccccc} 
& Años & CPA & Valor de $P$ & IC 95\% & Inflexión & IC 95\% \\
Tendencia 1 & $1983-1985$ & 86,92 & 0,000 & $38,8-151,7$ & - & $1985-1986$ \\
Tendencia 2 & $1985-1988$ & 317,09 & 0,000 & $209,8-461,6$ & 1985 & $1988-1989$ \\
Tendencia 3 & $1988-1997$ & 20,38 & 0,000 & $-20,1-7,6$ & 1989 & $1991-2000$ \\
Tendencia 4 & $1997-2001$ & $-7,30$ & 0,299 & $-12,1-59,4$ & 2001 & $1994-2013$ \\
Tendencia 5 & $2001-2004$ & 18,38 & 0,249 & $-3,9--1,2$ & 2004 & - \\
Tendencia 6 & $2004-2018$ & $-2,60$ & 0,000 & $17,3-27,5$ & - \\
AAPC & $1983-2018$ & 22,30 & 0,000 & & - \\
\hline
\end{tabular}

$\mathrm{VIH}$, virus de la inmunodeficiencia humana; CPA, cambio porcentual anual estimado; AAPC, cambio porcentual anual promedio para el período; IC95\%, intervalo de confianza al $95 \%$. Fuente: elaboración propia a partir de los resultados presentados en este estudio.

FIGURA 2. Casos observados de sida versus casos pronosticados mediante un modelo autorregresivo integrado de media móvil (ARIMA)

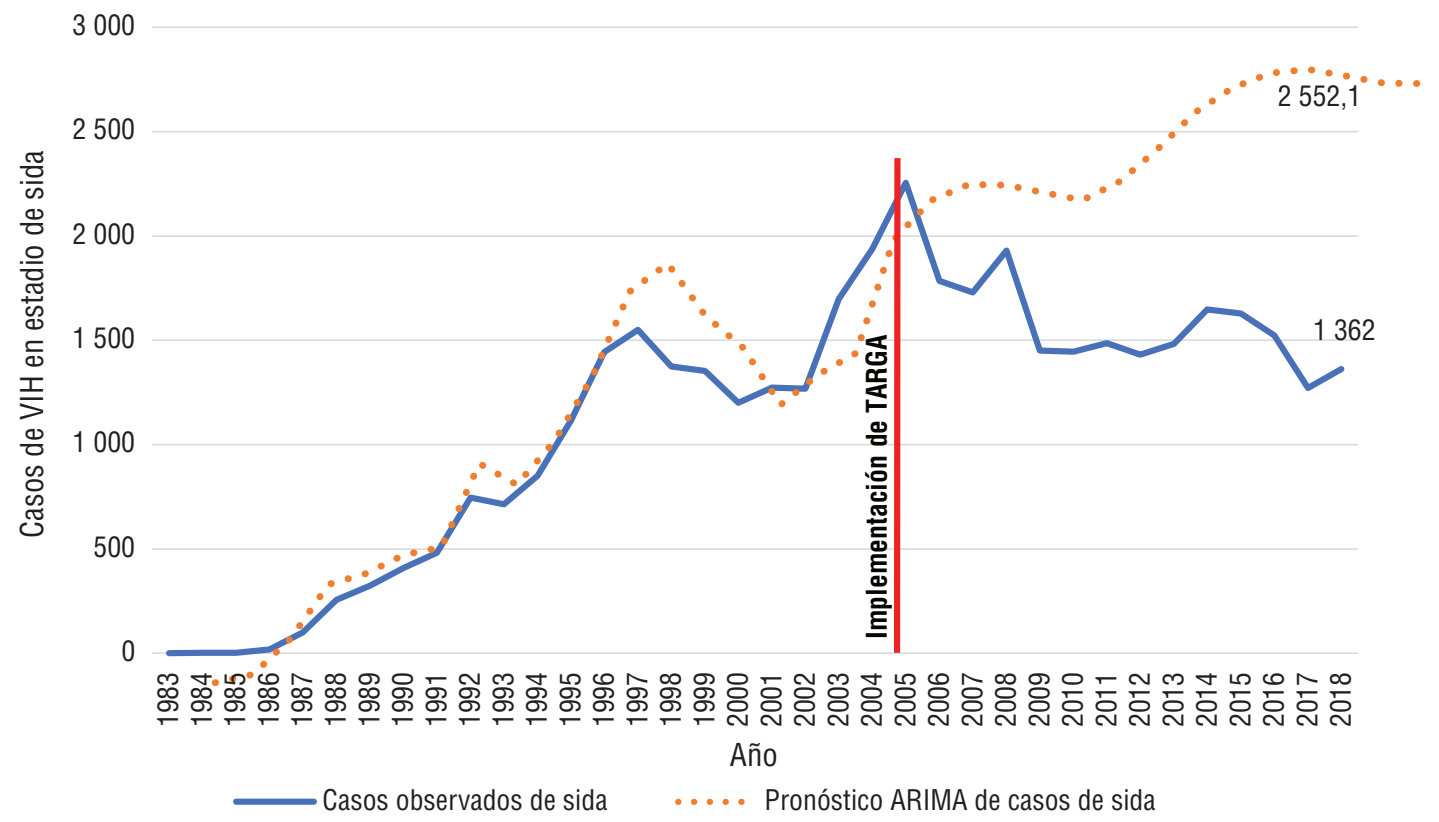

Fuente: elaboración propia a partir de los resultados del estudio

de pendiente indican ese incremento o decremento por año. En este sentido, aumentó de casi 90 casos de sida por cada año precedente al 2004 (89,98; IC 95\%: 72,17 - 107,80). Por otra parte, por cada año transcurrido posterior a su introducción ocurrió una disminución de 134,21 casos (IC 95\%: -160 - -108,60). De la misma manera, los resultados indicaron el efecto continuado del programa de una disminución anual de 44,4 casos de sida (IC 95\%: -62,84--25,96).

\section{DISCUSIÓN}

Los hallazgos del estudio evidencian que el Programa TARGA es una intervención efectiva del SSP en la prevención del número de casos de sida. En particular, permite el acceso universal a TARV y otorga otros servicios a las PVVS. La predicción realizada con el modelo ARIMA señaló, en los casos reportados de VIH estadio sida versus los casos pronosticados, un decremento de 45,8\% como resultado estimado de la actividad del programa. Además, la evidencia del impacto continúa en los
14 años posteriores a su introducción, lo cual es corroborado por la STI, que abarca todo el período posintroducción. En este sentido, se identificó un decremento persistente del número de casos anuales reportados con posterioridad a la implementación de TARGA. Este último patrón decreciente fue confirmado por la regresión joinpoint y forma parte de la visión general de 36 años que aporta el estudio en las tendencias de la detección de casos de sida en Perú.

Los resultados de la STI y de la predicción con ARIMA confirman que el Programa tuvo un efecto positivo en la disminución de casos nuevos de sida. Un estudio brasileño (24) confirma la eficacia de la introducción del tratamiento universal, con $60 \%$ en la disminución de casos de sida. Este cambio se debe, sobre todo, a que la TARV conduce a la reconstitución inmune en los pacientes infectados por el VIH y evita así la progresión al estadio sida (24-26).

El impacto del Programa TARGA en el análisis de STI reveló que, por cada año transcurrido posterior a su introducción, ocurrió una disminución sustancial en los casos nuevos de 
FIGURA 3. Serie de tiempo interrumpida para evaluar el efecto de TARGA (2004)

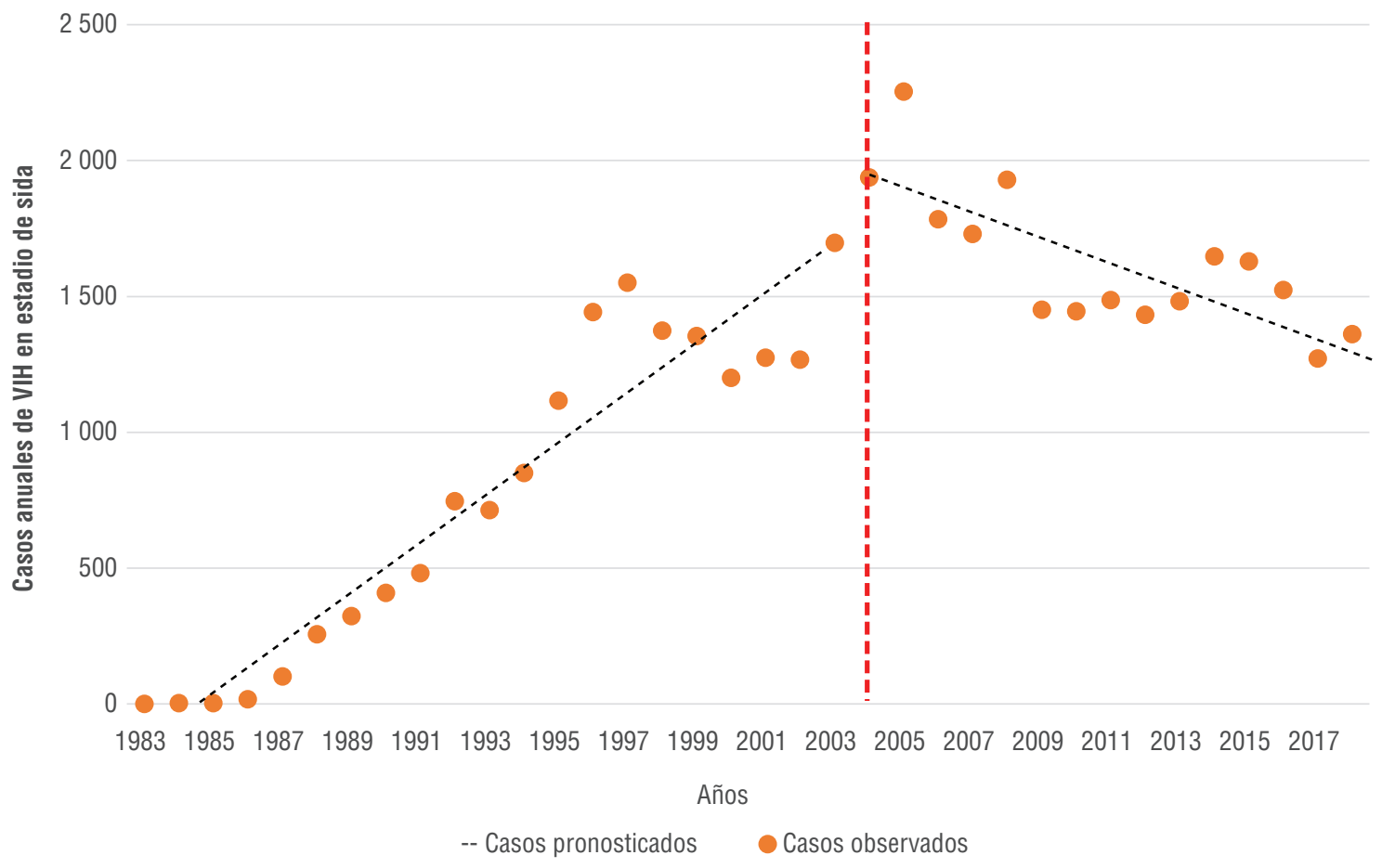

Fuente: elaboración propia a partir de los resultados del estudio

CUADRO 2. Impacto de la introducción del Programa TARGA en el sistema de salud peruano en la disminución de casos de sida (1983-2018), análisis de series de tiempo interrumpidas

\begin{tabular}{|c|c|c|c|c|c|c|c|c|c|c|c|c|}
\hline & \multicolumn{3}{|c|}{ 1983-2004 } & \multicolumn{3}{|c|}{2004} & \multicolumn{3}{|c|}{$2005-2018$} & \multicolumn{3}{|c|}{ Posestimación } \\
\hline & $\beta_{1}$ & IC 95\% & Valor de $P$ & $\beta_{2}$ & IC 95\% & Valor de $P$ & $\beta_{3}$ & IC 95\% & Valor de $\mathrm{P}$ & $\beta_{4}$ & IC 95\% & Valor de $P$ \\
\hline $\begin{array}{l}\text { Casos de VIH } \\
\text { estadio sida }\end{array}$ & 89,98 & $72,17-107,80$ & 0,000 & 174,75 & $-164,13-513,64$ & 0,30 & $-134,21$ & $-160,17--108,60$ & 0,000 & $-44,4$ & $-62,84--25,96$ & 0,000 \\
\hline
\end{tabular}

$\beta_{1}$ pendiente antes de la intervención; $\beta_{2}$ cambio de nivel en el período inmediatamente posterior al inicio de la intervención; $\beta_{3}$ término de interacción entre el tiempo desde el inicio del estudio y la variable que representa los períodos preintervención y posintervención indicando efecto en el tiempo; $\beta_{4}$ tendencia calculada del modelo mediante la combinación lineal de coeficientes $\left(\beta_{1}+\beta_{3}\right)$.

VIH, virus de la inmunodeficiencia humana.

Fuente: elaboración propia a partir de los resultados presentados en este estudio.

sida notificados. Un aspecto relevante en esta disminución es la modificación paulatina de los criterios para acceder a TARV. La primera norma técnica peruana (2004) estableció como criterio tener un CD4 inferior a 200 células $/ \mathrm{mm}^{3}$ (13). Sin embargo, se ha ido adelantando su comienzo con cortes cada vez más altos de CD4 ( 350 y 500 células $/ \mathrm{mm}^{3}$ ) como se recomendó luego $(17,18)$ a sabiendas de que el tratamiento universal reduce el riesgo de progresión de la infección (15). Esta forma temprana de tratamiento es el principal impulsor de la reducción de la morbilidad y la mortalidad por sida $(16,17,19)$. La actual norma peruana señala que se recomienda el inicio del tratamiento cualquiera sea el estadio clínico (18). No obstante, se deberá evaluar el impacto de esta recomendación, ya que su implementación y aceptabilidad requieren mayor tiempo (27).

De la misma manera, el número de casos anuales de sida experimentó una caída significativa y persistente, así como una disminución relevante en la tendencia durante los 14 años posteriores a su implementación. La introducción del Programa TARGA aún tiene un impacto significativo en el número de casos de sida notificados. En este sentido, a nivel nacional la cobertura del tratamiento se ha expandido de 5\% en el 2005 a $67 \%$ en el 2017 con un crecimiento exponencial en 11 años (28) y un aumento sustancial en el porcentaje de PVVS que reciben TARV. La cobertura estimada en Perú es comparable al promedio regional de otros países como Argentina (66\%), Brasil (64\%) y México (62\%) (29). Sin embargo, es superior a Bolivia (36\%), Guatemala (39\%) y El Salvador (48\%) (29). Es probable que estas divergencias sean causadas por disparidades en el acceso, retención y características nacionales de los programas (30). Por ejemplo, en Perú durante el 2014 la cobertura a nivel nacional fue de $46 \%$ y se incrementó a $73 \%$ en el $2018(9,29)$. En esta línea, la cantidad de establecimientos de salud encargados de realizar distribución de TARV aumentó de 86 (2011) a $152(2018)(9,31)$. Aunque la sociedad civil ha jugado un papel crucial para expandir el acceso universal (11), el estigma asociado al VIH/sida es una barrera importante que afecta el acceso a los servicios de salud y la adherencia a la medicación (32). 
Entre 1983 y 1997, se identificaron tres pendientes significativas de crecimiento acelerado. Otros estudios latinoamericanos con base poblacional también señalan una tendencia al crecimiento acelerado en el número de casos de sida notificados cada año $(33,34)$. De esta manera, un estudio chileno confirma un aumento de las tasas entre 2,6 y 3,5/100 000 habitantes a partir de 1992, hasta 6,0 en 2003 (33). De la misma forma, en Brasil se reportó un aumento con tasas de 8/100 000 habitantes en 1991, hasta 13,7 en 1998 (34).

Se observó que el cambio de nivel en la pendiente de casos en el período inmediatamente posterior al inicio del Programa TARGA no resultó significativo. Luego, se destaca un incremento en los casos de sida del 2005. Esto pudo deberse a que el presupuesto del gobierno peruano dedicado a la lucha contra el VIH/sida no fue suficiente (11). De hecho, en el año 2004, se destinó $24 \%$ del presupuesto para antirretrovirales; mientras que durante el 2005 estos no se compraron. Sin embargo, en el 2006 se triplicó el presupuesto, y se destinó 56\% para la compra de TARV, lo que se tradujo en 7000 tratamientos para 15 meses (11).

Son diversas las fortalezas del programa TARGA para lograr la disminución de casos de sida. En primer lugar, la incorporación de la investigación basada en evidencias dentro de sus líneas de acción. Esto se ha reflejado, por ejemplo, en la disminución progresiva del nivel de recuento de linfocitos CD4 para acceder al tratamiento hasta llegar al actual acceso universal (18). En segundo lugar, el compromiso del estado para hacerse responsable del control total de la enfermedad luego de haber recibido el apoyo de financiadores internacionales. Este factor ha logrado aumentar, en forma paulatina, la compra de medicamentos y la implementación de establecimientos de salud con profesionales entrenados donde se otorga el tratamiento de manera gratuita $(9,11)$.

Otra fortaleza es la descentralización de la toma de muestras y de los establecimientos donde se brinda el tratamiento. Si bien los exámenes de CD4 y carga viral los realiza el Instituto Nacional de Salud del Ministerio de Salud (MINSA), la toma de muestras se realiza en todos los establecimientos que ofrecen TARV, donde existe personal encargado de trasladarlas $(9,18)$. Además, el MINSA ha realizado convenios con importantes centros de salud privados que trabajan con población clave para otorgar el tratamiento. En el 2017, 10,6\% de las personas que recibieron TARV fueron atendidas en estos centros (31).

Un cuarto punto fuerte es la capacitación y sensibilización del personal de salud. El estigma en contra del VIH/sida es una barrera que aún existe y es más dañina cuando proviene del personal de salud (32). Por último, el uso de tecnologías de la información ha jugado un papel importante. En el 2007, el MINSA implementó la plataforma web "NETLAB", que permite a los médicos tratantes ver los resultados del recuento de linfocitos CD4 y carga viral vía Internet (35). Esto agiliza la toma de decisiones para la atención oportuna de los pacientes (35).

Como limitaciones del estudio, no es posible excluir el subregistro de casos, ya que en Perú un número significativo de sujetos infectados desconocen su estado (36\%) (20). No fueron abordados determinantes de la variable desenlace, lo que se debe tener en consideración al generalizar los hallazgos. Sin embargo, esta limitación fue minimizada al controlar la presencia de variables contextuales importantes; por ejemplo, la presencia de otros programas que pudieran afectar la validez externa y mediante el empleo del diseño cuasiexperimental con el análisis de STI (22). Entre las limitaciones inherentes de este diseño se señala que, debido a la ausencia de datos, no fue posible incluir un grupo de comparación, lo que ofrecería mayores evidencias en el verificar el efecto del Programa. De la misma manera, se debe indicar que en el período estudiado ocurrieron cambios respecto a los criterios diagnósticos del estadio sida, lo cual puede tener relación con el subregistro de casos. A pesar de las limitaciones, la mayor fortaleza del presente análisis es ser el primero en modelar y predecir el impacto del Programa TARGA considerando un período de 36 años. Además, las estimaciones realizadas podrían ser utilizadas para un estudio de costo-efectividad de su aplicación.

Los hallazgos evidencian un aumento sustancial en los casos de sida durante el período 1983-2004, con una caída significativa y repentina luego del 2005. La disminución en los casos reportados de sida se relaciona con la introducción del Programa TARGA y su paulatino incremento en la cobertura. El análisis de series de tiempo interrumpidas y la predicción con ARIMA han permitido determinar el impacto positivo y continuado en el tiempo del Programa desde su introducción en el 2004.

El Perú debe continuar con la expansión de la cobertura de la TARV hasta alcanzar su acceso universal. No obstante, la ampliación en la cobertura se deberían garantizar iguales condiciones de acceso en las diversas regiones del país para las PVVS. La disponibilidad de la medicación apenas realizado el diagnóstico de VIH contribuiría en la reducción de los casos nuevos de sida.

Contribución de los autores. Todos los autores YEC, GDG y LMA concibieron el estudio original, recolectaron y analizaron los datos, interpretaron los resultados y escribieron y revisaron el manuscrito. Todos los autores revisaron y aprobaron la versión final.

Conflicto de intereses. Ninguno declarado por los autores.

Declaración. Las opiniones expresadas en este manuscrito son responsabilidad del autor y no reflejan necesariamente los criterios ni la política de la RPSP/PAJPH y / o de la OPS.

\section{REFERENCIAS}

1. Chow EP, Grulich AE, Fairley CK. Epidemiology and prevention of sexually transmitted infections in men who have sex with men at risk of HIV. The Lancet HIV. 2019;6(6):396-405. doi: 10.1016/ S2352-3018(19)30043-8.

2. Sued O, Figueroa MI, Cahn P. Clinical challenges in HIV/AIDS: Hints for advancing prevention and patient management strategies.
Adv Drug Deliv Rev. 2016;103:5-19. doi:10.1016/j.addr.2016. 04.016.

3. Iskarpatyoti BS, Lebov J, Hart L, Thomas J, Mandal M. Evaluations of Structural Interventions for HIV Prevention: A Review of Approaches and Methods. AIDS Behav. 2018;22(4):1253-1264. doi:10.1007/ s10461-017-1997-x. 
4. Becerra JC, Bildstein LS, Gach JS. Recent Insights into the HIV/ AIDS Pandemic. Microb Cell. 2016;3(9):451-475. Published 2016 Sep 5. doi:10.15698/mic2016.09.529.

5. Cohen MS, Chen YQ, McCauley M, Gamble T, Hosseinipour MC, Kumarasamy N, Hakim JG, Kumwenda J, Grinsztejn B, Pilotto JH, Godbole SV. Antiretroviral therapy for the prevention of HIV-1 transmission. N Engl J Med. 2011;365(6):493-505. doi:10.1056/ NEJMoa110524.

6. Caro-Vega Y, Belaunzarán-Zamudio PF, Crabtree-Ramírez BE, Shepherd BE, Grinsztejn B, Wolff M, Pape JW, Padgett D, Gotuzzo E, McGowan CC, Sierra-Madero JG. Durability of efavirenz compared with boosted protease inhibitor-based regimens in antiretroviral-naïve patients in the Caribbean and Central and South America. Open Forum Infect Dis. 2018;5(3):ofy004. Published 2018 Mar 2. doi:10.1093/ofid/ofy004.

7. Günthard HF, Aberg JA, Eron JJ, Hoy JF, Telenti A, Benson CA, et al. Antiretroviral treatment of adult HIV infection: 2014 recommendations of the International Antiviral Society-USA Panel. Jama. 2014;312(4):410-25. doi:10.1001/jama.2014.8722.

8. Indexmundi [en Internet]. Charlotte: Habitantes infectados con VIH/SIDA - Sudamérica; c2019 Disponible en: https://www. indexmundi.com $/ \mathrm{map} / ? \mathrm{t}=0 \& \mathrm{v}=35 \& \mathrm{r}=\mathrm{sa} \& \mathrm{l}=\mathrm{es}$. Acceso el 30 de septiembre de 2019.

9. Centro Nacional de Epidemiología, Prevención y Control de Enfermedades. Situación epidemiológica del VIH-Sida en el PerúBoletín mensual 2018 [en Internet]. Lima: CDC; 2018. Disponible en:http://www.dge.gob.pe/portal/docs/vigilancia/vih/Boletin_2018/diciembre.pdf. Acceso el 30 de septiembre de 2019.

10. UNAIDS [en Internet]. Ginebra: Perú. Visión general. c2019. Disponible en: https://www.unaids.org/es/regionscountries/countries/ peru. Acceso el 30 de septiembre de 2019.

11. MINSA. Los primeros dos años de Acceso Universal al tratamiento antirretroviral en el Perú [en Internet]. Lima: MINSA; 2006. Disponible en: http://bvs.minsa.gob.pe/local/dgsp/148_\%20ATR1.pdf. Acceso el 30 de septiembre de 2019.

12. Eduardo Gotuzzo H. SIDA en el Perú: Impacto de la terapia antirretroviral de gran actividad (TARGA). Rev Med Hered. 2007;18(4):181-3.

13. MINSA. Norma técnica para el tratamiento antirretroviral de gran actividad (TARGA) en adultos infectados por VIH [en Internet]. Lima: MINSA; 2004. en: https://www.who.int/hiv/pub/guidelines/peru_art.pdf. Acceso el 30 de septiembre de 2019.

14. Nelson AM, Manabe YC, Lucas SB. Immune reconstitution inflammatory syndrome (IRIS): what pathologists should know. Semin Diagn Pathol. 2017;34(4):340-351. doi:10.1053/j.semdp.2017.04.010.

15. Lundgren JD, Borges AH, Neaton JD. Serious Non-AIDS Conditions in HIV: Benefit of Early ART. Curr HIV/AIDS Rep. 2018;15(2):162-171.

16. INSIGHT START Study Group, Lundgren JD, Babiker AG, Gordin F, Emery S, Grund B, et al. Initiation of Antiretroviral Therapy in Early Asymptomatic HIV Infection. N Engl J Med. 2015;373(9):795-807. doi:10.1056/NEJMoa1506816.

17. World Health Organization. Consolidated guidelines on the use of antiretroviral drugs for treating and preventing HIV infection. Recommendations for a public health approach [in Internet]. Geneva: WHO; 2016. Disponible en https://www.who.int/hiv/ pub/arv/arv-2016/en/. Acceso el 30 de septiembre de 2019

18. MINSA. Norma técnica de salud de atención integral del adulto con infección por el VIH [en Internet]. Lima: MINSA; 2018. Disponible en: http://bvs.minsa.gob.pe/local/MINSA/4479.pdf. Acceso el 30 de septiembre de 2019.
19. Ruiz MS, O'Rourke A, Allen ST. Impact Evaluation of a Policy Intervention for HIV Prevention in Washington, DC. AIDS Behav. 2016;20(1):22-28. doi:10.1007/s10461-015-1143-6.

20. Garcia-Fernandez L, Novoa R, Huaman B, Benites C. Continuum of care of people living with HIV and gaps in achieving the 90-90-90 goals in Peru. Rev Peru Med Exp Salud Publica. 2018;35(3):491-496.

21. Leatherdale ST. Natural experiment methodology for research: a review of how different methods can support real-world research. International Journal of Social Research Methodology. 2019;22(1):19-35.

22. Bernal JL, Cummins S, Gasparrini A. Interrupted time series regression for the evaluation of public health interventions: a tutorial. Int J Epidemiol. 2017;46(1):348-355. doi:10.1093/ije/dyw098.

23. León-Álvarez AL, Betancur-Gómez JI, Jaimes-Barragán F, Grisales-Romero H. Clinical and epidemiological rounds. Time series. Iatreia. 2016;29(3):373-81.

24. Pereira GF, Sabidó M, Caruso A, Benzaken AS. Decline in reported AIDS cases in Brazil after implementation of the test and treat initiative. BMC Infect Dis. 2019;19(1):579. doi:10.1186/s12879-019-4018-z.

25. Cain LE, Logan R, Robins JM, et al. When to initiate combined antiretroviral therapy to reduce mortality and AIDS-defining illness in HIV-infected persons in developed countries: an observational study. Ann Intern Med 2011;154:509-515.

26. Cohen MS, Chen YQ, McCauley M, Gamble T, Hosseinipour MC, Kumarasamy N, et al. Prevention of HIV-1 infection with early antiretroviral therapy. N Engl J Med. 2011;365(6):493-505. doi:10.1056/ NEJMoa1105243.

27. Stranix-Chibanda L, Brummel S, Pilotto J, Mutambanengwe M, Chanaiwa V, Mhembere T, et al. Slow Acceptance of Universal Antiretroviral Therapy (ART) Among Mothers Enrolled in IMPAACT PROMISE Studies Across the Globe. AIDS Behav. 2019;23(9):25222531. doi:10.1007/s10461-019-02624-3.

28. Indexmundi [en Internet]. Charlotte: Peru - Antiretroviral therapy coverage; c2019. Disponible en: https://www.indexmundi.com/ facts/peru/indicator/SH.HIV.ARTC.ZS. Acceso el 30 de septiembre de 2019.

29. The World Bank [in Internet]. Washington: Antiretroviral therapy coverage; c2019. Disponible en: https://data.worldbank.org/indicator/SH.HIV.ARTC.ZS. Acceso el 30 de septiembre de 2019.

30. UNAIDS. 90-90-90. On the right track towards the global target [en Internet]. Ginebra: UNAIDS; 2016 Disponible en: https:/ / reliefweb. int/sites/reliefweb.int/files/resources/90_90_90_Progress_ReportFINAL.pdf. Acceso el 30 de septiembre de 2019.

31. MINSA. Situación Actual del VIH-SIDA en el Perú [en Internet] Lima: MINSA; 2018. Disponible en: http://www.digemid.minsa. gob.pe/UpLoad/UpLoaded/PDF/EAccMed/ReunionesTecnicas/ PONENCIAS/2018/DIA3/SituacionActualVIH-SIDA.pdf. Acceso el 30 de septiembre de 2019.

32. Zafra-Tanaka JH, Ticona-Chavez E. Stigma related to HIV/AIDS associated with adherence to antiretroviral therapy in patients of a public hospital in Lima, Peru 2014. Rev Peru Med Exp Salud Publica. 2016;33(4):625-632.

33. García M, Olea A. Evolución y situación epidemiológica de la infección por virus de inmunodeficiencia humana y síndrome de inmunodeficiencia adquirida en Chile. Rev Chil Infect. 2008;25(3):162-70.

34. Brito Ana Maria de, Castilho Euclides Ayres de, Szwarcwald Célia Landmann. AIDS e infecção pelo HIV no Brasil: uma epidemia multifacetada. Rev. Soc. Bras. Med. Trop. 2001;34(2): 207-217.

35. Vargas-Herrera J, Segovia-Juarez J, Garro Nuñez GM. Sistema de información de la red nacional de laboratorios de salud pública en el Perú (Netlab. Rev Peru Med Exp Salud Publica 2015;32(2):378-84. 


\section{Impact of the TARGA Program on the reduction of AIDS cases in the Peruvian health system, 1983-2018}

ABSTRACT Objective. To evaluate the impact of the Highly Active Antiretroviral Therapy (HAART) Program in the reduction of AIDS stage cases in Peru between 1983-2018.

Methods. Natural experiment with interrupted time series design considering a period of 36 years. Cases of AIDS were analyzed according to the year of diagnosis and the policy of universal access to antiretroviral therapy of 2004. Aggregated data from the National Center for Epidemiology, Prevention and Control of Diseases from 1983 to 2018 were used. Linear logarithmic regression was used and the effect of the HAART Program was evaluated with an autoregressive integrated moving average (ARIMA) model to make forecasting of the expected number of annual cases of AIDS, and through interrupted time series.

Results. Between 2004-2018 there was an annual decrease of 2.60\% (95\% Cl:-3.9-1.2) of cases. The best identified ARIMA model had the components: $(8,3,1)$. The forecasting yielded a total of 32709 new cases of AIDS for this period, indicating the estimated prevention of 10280 cases. The time-series analysis showed an annual decrease of 134.21 (95\% Cl:-160- -108.60) cases.

Conclusions. The findings showed a substantial increase in AIDS cases during the period 1983-2004, with a significant drop in cases after the implementation of the HAART Program. The Program is an effective intervention for the prevention of the number of AIDS cases, allowing universal access to antiretroviral therapy and providing other services such as monitoring tests, psychological counseling and the promotion of adherence to treatment in people living with HIV and AIDS.

Keywords Health policy; antiretroviral therapy, highly active; acquired immunodeficiency syndrome; health systems; Peru.

\section{Impacto do Programa HAART na redução de casos de AIDS no sistema de saúde peruano,1983-2018}

RESUMO Objetivo. Avaliar o impacto do Programa de Terapia Antirretroviral Altamente Ativa (HAART) na redução de casos em fase de Aids no Peru de 1983 a 2018.

Métodos. Experimento natural com desenho de séries temporais interrompidas considerando um período de 36 anos. Os casos de Aids foram analisados de acordo com o ano de diagnóstico e a política de acesso universal à terapia antirretroviral em 2004. Foram utilizados dados agregados do Centro Nacional de Epidemiologia, Prevenção e Controle de Doenças de 1983 a 2018. Utilizou-se regressão logarítmica linear. O efeito do HAART foi avaliado utilizando um modelo de autorregressão de média móvel integrada (ARIMA) para prever o número esperado de casos anuais de Aids; e através de séries temporais interrompidas.

Resultados. De 2004 a 2018 houve uma diminuição anual de 2,60\% (95\% Cl: -3,9 a 1,2) nos casos. Com base no melhor modelo ARIMA, que continha os componentes (8,3,1), foram previstos 32709 casos novos de Aids para o período, indicando uma prevenção estimada de 10280 casos. A análise da série temporal mostrou uma diminuição anual de 134,21 casos (95\%Cl: -160 a -108,60).

Conclusões. Os resultados mostraram um aumento substancial de casos de Aids durante o período de 1983 a 2004, com queda significativa nos casos após a implementação do HAART. O HAART é uma intervenção eficaz para a prevenção de casos de Aids, permitindo o acesso universal à terapia antirretroviral e envolvendo outros serviços como testes de monitorização, aconselhamento psicológico e promoção da adesão ao tratamento em pessoas vivendo com HIV e Aids.

Palavras-chave Política de saúde; terapia antirretroviral de alta atividade; síndrome de imunodeficiência adquirida; sistemas de saúde; Peru. 